\title{
Flow of Viscoelastic Fluid in a Curved Pipe
}

\author{
Part 1: Velocity Distribution of a Flow in a Toroidal Pipe of Circular Cross-Section \\ By Yoshiyuki Iemoto and Kichidayu Sawazaki, Members, TMSJ \\ Faculty of Engineering, Fukui University, Fukui
}

Based on the Journal of the Textile Machinery Society of Japan, Transactions, Vol. 34, No. 1, T1-T11 (1981-1)

\begin{abstract}
The steady flow of viscoelastic fluid in a toroidal pipe of circular cross-section is analytically solved with the White-Metzner constitutive equation by a perturbation method. The results are as follows:

(1) The flow rate of a secondary flow in the cross-sectional plane increases with decreasing the viscosity index $n$, and with increasing the elasticity index $s$, the Reynolds number $R e$ and the Weissenberg number $W e$.

(2) The position of the maximum axial flow velocity shifts on the central plane more outward from the pipe center for smaller $n$ and larger $R e$ in the case of Power law fluid, and for larger $s$ in the case of White-Metzner fluid.

(3) Owing to the centrifugal and elastic forces applied on the fluid, the stream line starting at a pipe center shifts outward on the central plane. The larger the values of $s, R e$ and $W e$ are, the larger the amount of the outward shift is.

Hence, the effects of $R e$ and $W e$ on the flow are shown to be similar to an available experimental result as well as earlier theoretical results. Also, it is found that the values $n$ and $s$, which represent the degrees of decrement of the fluid viscosity and the first normal stress coefficient with the shear-rate respectively, have significant effects on the flow at high shear-rate to which the earlier analyses could not be applied.
\end{abstract}

\section{Introduction}

The steady flow of viscoelastic fluid such as polymer melts or solutions in a curved pipe is important because it is a fundamental phenomenon in textile, high polymer, mechanical industries and so on. For a Newtonian fluid, Dean $^{[1-2]}$ firstly analyzed a laminar flow in a toroidal pipe of circular cross-section with small radius-ratio (ratio of radii of pipe curvature to pipe cross-section). The toroidal pipe is one of the most basic configurations among curved pipes. Nowadays the flow in a helically coiled pipe is theoretically solved ${ }^{[3]}$. On the other hand, the laminar flow of non-Newtonian fluid in a toroidal pipe of circular crosssection with small radius-ratio was analyzed by Jones ${ }^{[4]}$ for Reiner-Rivlin-Prager model, by Clegg and Power ${ }^{[5]}$ for Bingham model, by Thomas and Walters ${ }^{[6]}$ for OldroydWalters-Fredrickson model, and by Jones ${ }^{[7]}$ for ColemanNoll's third order fluid which contains Reiner-Rivlin-Prager fluid and Oldroyd-Walters-Fredrickson model as its special case. In the analyses the perturbation method that a straight pipe flow is a zeroth order approximation was applied. Namely, Jones ${ }^{[4]}$, Clegg and Power ${ }^{[5]}$ expanded by applying an exact method ${ }^{[1]}$ for Newtonian fluid. Thomas and
Walters $^{[6]}$, Jones ${ }^{[7]}$ applied an approximate method ${ }^{[2]}$ for relatively large Reynolds number. They showed that two symmetric spirals with respect to the central plane containing the center axis of the curved pipe appear in the Bingham fluid as well as in Newtonian fluid, but the stream line of secondary flow is significantly different from that of Newtonian fluid because a plug flow is in the vicinity of the center axis. For the viscoelastic fluids it is found that two symmetric spirals appear as well as in the viscous fluid, but that the flow rate of secondary flow is larger and the axial pitch of spirals is shorter than in the viscous fluid ${ }^{[4,6,7]}$. These predictions agreed qualitatively with experiments by Barnes and Walters ${ }^{[7]}$. However, the distributions of axial flow velocities of Reiner-Rivlin-Prager fluid and OrdroydWalters-Fredrickson model in a straight pipe of circular cross-section do not show non-Newtonian viscosity, but show Poiseuille distributions. The Coleman-Noll's third order fluid can not be applied to the industrially useful flow at high shear-rate where the viscosity and the normal stress coefficient decrease with the increment of shear-rate, because its normal stress coefficient is constant independent of the shear-rate in a simple shear flow. 
The viscosity of real fluids is finite at zero shear-rate, but de Waele-Ostwald fluid (Power law fluid) has such defects as its viscosity becomes infinity at zero shear-rate for pseudoplastic fluid. Also, it is difficult in most cases to solve governing equations, because the constitutive equation is nonlinear. However, de Waele-Ostwald fluid shows non-Newtonian viscosity in a flow through a straight pipe of circular crosssection, and from this usefulness it is often applied to this flow. So, it is thought that the White-Metzner mode ${ }^{[8]}$ used by Denn ${ }^{[9]}$, the viscosity and the first normal stress coefficient of which are obedient to a power law for shear-rate, is useful to analyze a flow of viscoelastic fluid at relatively high shearrate that is industrially necessary.

Hence, the present paper deals with the steady flow of incompressible viscoelastic fluid in a toroidal pipe of circular cross-section with small radius-ratio, by the use of the White-Metzner constitutive equation ${ }^{[8]}$.

\section{Coordinate System and Fundamental Equations}

\subsection{Coordinate system}

The coordinate system shown in Fig. 1 is used in the analysis. The center axis of a curved pipe of circular crosssection of radius $a$ is on the $x y$ plane of Cartesian coordinate system $(x, y, z)$, and the point $C$ is the cross-sectional center of the curved pipe cut by a plane that makes an angle $x^{3}$ with $x z$ plane. The curvature center of the curved pipe is at the origin $\mathrm{O}$ of Cartesian coordinate system and the radius of curvature of the pipe is denoted by $R^{*}$. The position of a point $\mathbf{P}$ inside the pipe is specified by the orthogonal curvilinear coordinate system $\left(x^{1}, x^{2}, x^{3}\right)$, where $x^{1}$ is a radial position from the pipe center, $x^{2}$ an angle made with a line parallel to $z$ axis through the point $C$ by the line CP. Then, the orthogonal curvilinear coordinate system $\left(x^{1}, x^{2}, x^{3}\right)$ is related to Cartesian coordinate system $(x, y, z)$ as follows:

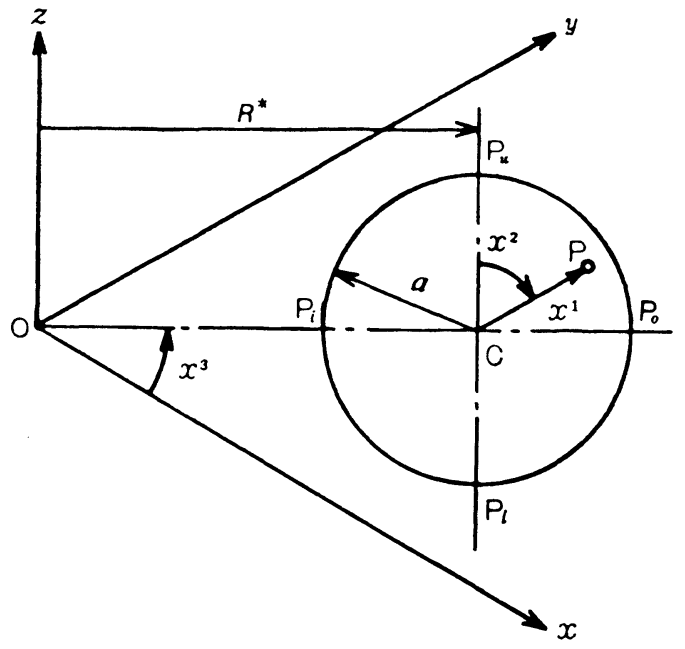

Fig. 1 Coordinate system

$$
\begin{aligned}
& x=\left(R^{*}+x^{1} \sin x^{2}\right) \cos x^{3} \\
& y=\left(R^{*}+x^{1} \sin x^{2}\right) \sin x^{3} \\
& z=x^{1} \cos x^{2}
\end{aligned}
$$

and the metric tensor is

$$
\left.\begin{array}{l}
g_{11}=1, \quad g_{22}=\left(x^{1}\right)^{2}, \quad g_{33}=\left(R^{*}+x^{1} \sin x^{2}\right)^{2} \\
g_{12}-g_{21}=g_{13}=g_{31}=g_{23}=g_{32}=0
\end{array}\right\} \cdots(2)
$$

\subsection{Fundamental equations}

Cauchy's equation of motion and the equation of continuity governing the steady flow of incompressible fluid in a curved pipe under a constant pressure gradient in the flow direction are written in tensor forms

$$
\begin{aligned}
& \rho v^{j} v^{i},{ }_{j}=-p^{*},{ }_{j} g^{i j}+p^{i j},{ }_{j} \\
& v^{j},{ }_{j}=0 \\
& \cdots \ldots \ldots \ldots \ldots \ldots \ldots \ldots \ldots \ldots \ldots \ldots \ldots \ldots
\end{aligned}
$$

where $\rho$ is the fluid density, $v^{j}$ the flow velocity vector, $p^{*}$ the isotropic pressure and $p^{i j}$ the deviatric stress tensor. The following White-Metzner model ${ }^{[8]}$ is applied to constitutive equation:

$$
p^{i j}+\lambda\left(4 \mid \mathrm{I}\left[e^{*} \mid\right)^{s-n-1} \delta p^{i j} \begin{array}{c}
\delta \\
\delta
\end{array} \quad-2 \eta\left(4||\left[e^{*} \mid\right)^{n-1}{ }^{2} e^{i j}\right.\right.
$$

where $\lambda, \eta, s$ and $n$ are material constants and $s$ and $n$ are named as the elasticity index and the viscosity index, respectively. The elasticity index $s$ is between about 1 and 2, the viscosity index $n$ between 0.2 and $1^{[9]}$ and ordinarily $s>n^{[10]}$. Eq. (5) represents Maxwell model in case that $s=2$ and $n=1$, Power law fluid in case that $\lambda=0$ and Newtonian fluid in case that $\lambda=0$ and $n=1$. The notation $e^{i j}$ is the rate-of-deformation tensor, II $e^{*}$ the second invariant of rateof-deformation tensor and $\delta p^{i j} / \delta t$ the Oldroyd time derivative for deviatric stress tensor. They are given by

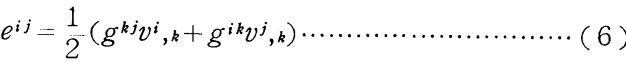

$$
\begin{aligned}
& 4\left|\Pi e^{*}\right|=2\left[\left(e^{1}\right)^{2}+\left(e^{2_{2}}\right)^{2}+\left(e^{3_{3}}\right)^{2}\right] \\
& +4\left[e^{1} e^{2}{ }_{1}+e^{2}{ }_{3} e^{3}{ }_{2}+e^{3}{ }_{1} e^{1}{ }_{3}\right] \\
& \frac{\delta p^{i j}}{\delta t}=v^{k} p^{i j}{ }_{k}-v^{i}{ }_{k} p^{k j}-v^{j}{ }_{k} p^{i k}
\end{aligned}
$$

In eq. (5), $\lambda\left(4\left|\mathrm{II} e^{*}\right|\right)^{(s-n-1) / 2}$ is the relaxation time. The second term of its left-hand side represents an elastic stress, and $\eta\left(4\left|I I e^{*}\right|\right)^{(n-1) / 2}$ is the viscosity. The right-hand side represents a viscous stress.

After the above variables are converted to physical components, in order to analyze in non-dimensional variables, the lengthes are non-dimensionalized by the pipe radius $a$, the velocities being by the velocity component $W$ on the pipe center in $x^{3}$ direction, and the stresses being by $\rho W^{2}$ as follows: 


$$
\begin{aligned}
& R=R^{*} / a \\
& x_{1}=x^{1} / a, \quad x_{2}=x^{2}, \quad x_{3}=x^{3} \\
& v_{i}=\sqrt{g_{i i}} v^{i} / W \\
& p=p^{*} /\left(\rho W^{2}\right), p_{i j}=\sqrt{g_{i i}} \sqrt{g_{j j}} p^{i j} /\left(\rho W^{2}\right) \\
& I I_{e}=\amalg I e^{*} /(W / a)^{2} \\
& e_{i j}=\sqrt{g_{i i}} \sqrt{g_{j j}} e^{i j} /(W / a) \\
& \frac{\delta p}{\delta t_{i j}}=\sqrt{g_{i i}} \sqrt{g_{j j}} \frac{\delta p^{i j}}{\delta t} /\left(\rho W^{3} / a\right)
\end{aligned}
$$

where Einstein's summation convention is not applied. In eq. (9), $g_{i j}$ represents the covariant metric tensor, but $x_{i}$ is non-dimensional value of coordinate, and $v_{i}, p_{i j}, e_{i j}, \delta p / \delta t_{i j}$ are not covariant tensors but non-dimensional physical components. All variables with subscript in the followings represent non-dimensional ones. The velocity $W$ is approximately equal to the velocity at the center axis of the straight pipe as shown in later results.

Cauchy's equation of motion and the equation of continuity are written in non-dimensional forms

$$
\begin{gathered}
v_{1} \frac{\partial v_{1}}{\partial x_{1}}+\frac{v_{2}}{x_{1}} \frac{\partial v_{1}}{\partial x_{2}}-\frac{v_{2}^{2}}{x_{1}}-\frac{\sin x_{2} v_{3}^{2}}{h} \\
=-\frac{\partial p}{\partial x_{1}}+\frac{\partial p_{11}}{\partial x_{1}}+\left(\frac{1}{x_{1}}+\frac{\sin x_{2}}{h}\right) p_{11} \\
+\frac{1}{x_{1}} \frac{\partial p_{12}}{\partial x_{2}}+\frac{\cos x_{2}}{h} p_{12}-\frac{p_{22}}{x_{1}}-\frac{\sin x_{2}}{h} p_{33} \\
v_{1} \frac{\partial v_{2}}{\partial x_{1}}+\frac{v_{2}}{x_{1}} \frac{\partial v_{2}}{\partial x_{2}}+\frac{v_{1} v_{2}}{x_{1}}-\frac{\cos x_{2}}{h} v_{3}^{2} \\
=-\frac{1}{x_{1}} \frac{\partial p}{\partial x_{2}}+\frac{\partial p_{12}}{\partial x_{1}}+\left(\frac{2}{x_{1}}+\frac{\sin x_{2}}{h}\right) p_{12} \\
+\frac{1}{x_{1}} \frac{\partial p_{22}}{\partial x_{2}}+\frac{\cos x_{2}}{h} p_{22}-\frac{\cos x_{2}}{h} p_{33} \\
v_{1} \frac{\partial v_{3}}{\partial x_{1}}+\frac{v_{2}}{x_{1}} \frac{\partial v_{3}}{\partial x_{2}}+\frac{\sin x_{2}}{h} v_{1} v_{3}+\frac{\cos x_{2}}{h} v_{2} v_{3} \\
=-\frac{1}{h} \frac{\partial p}{\partial x_{3}}+\frac{\partial p_{13}}{\partial x_{1}}+\left(\frac{1}{x_{1}}+\frac{2 \sin x_{2}}{h}\right) p_{13} \\
\quad+\frac{1}{x_{1}} \frac{\partial p_{23}}{\partial x_{2}}+\frac{2 \cos x_{2}}{h} p_{23} \\
\frac{\partial v_{1}}{\partial x_{1}}+\frac{v_{1}}{x_{1}}+\frac{1}{x_{1}} \frac{\partial v_{2}}{\partial x_{2}}+\frac{\sin x_{2}}{h} v_{1}+\frac{\cos x_{2}}{h} v_{2}=0 \cdots
\end{gathered}
$$

where

$$
h=R+x_{1} \sin x_{2}
$$

because the flow is independent of $x_{3}$ except for the isotropic pressure $p$.

The constitutive equation is written as

$$
p_{i j}+W_{e} M^{s-n-1} \frac{\delta p}{\delta t_{i j}}=\frac{2}{R_{e}} M^{-\frac{n-1}{2}} e_{i j}
$$

where $W e$ is the Weissenberg number and $R e$ is the Reynolds number. They are defined as

$$
\begin{aligned}
& W_{e}=\lambda(W / a)^{s-n} \\
& R_{e}=\rho W^{2-n} a^{n} / \eta
\end{aligned}
$$

The variables $M, e_{i j}$ and $\delta p / \delta t_{i j}$ are

$$
\begin{aligned}
& M \equiv 4|I I .|=2\left(e_{11^{2}}+e_{22}{ }^{2}+e_{33^{2}}\right)+4\left(e_{12}{ }^{2}+e_{23^{2}}+e_{31^{2}}\right) \\
& e_{11}=\frac{\partial v_{1}}{\partial x_{1}}, \quad e_{22}=\frac{1}{x_{1}} \frac{\partial v_{2}}{\partial x_{2}}+\frac{v_{1}}{x_{1}} \\
& e_{33}=v_{1} \frac{\sin x_{2}}{h}+v_{2} \frac{\cos x_{2}}{h}, \quad e_{12}=\frac{1}{2}\left(\frac{1}{x_{1}} \frac{\partial v_{1}}{\partial x_{2}}+\frac{\partial v_{2}}{\partial x_{1}}-\frac{v_{2}}{x_{1}}\right) \\
& e_{23}=\frac{1}{2}\left(\frac{1}{x_{1}} \frac{\partial v_{3}}{\partial x_{2}}-v_{3} \frac{\cos x_{2}}{h}\right), \quad e_{13}=\frac{1}{2}\left(\frac{\partial v_{3}}{\partial x_{1}}-v_{3} \frac{\sin x_{2}}{h}\right) \\
& \frac{\delta p}{\delta t_{11}}=v_{1} \frac{\partial p_{11}}{\partial x_{1}}+\frac{v_{2}}{x_{1}} \frac{\partial p_{11}}{\partial x_{2}}-2\left(p_{11} \frac{\partial v_{1}}{\partial x_{1}}+\frac{p_{12}}{x_{1}} \frac{\partial v_{1}}{\partial x_{2}}\right) \\
& \frac{\delta p}{\delta t_{22}}=v_{1} \frac{\partial p_{22}}{\partial x_{1}}+\frac{v_{2}}{x_{1}} \frac{\partial p_{22}}{\partial x_{2}} \\
& -2\left(\frac{v_{1}}{x_{1}} p_{22}-\frac{v_{2}}{x_{1}} p_{12}+\frac{\partial v_{2}}{\partial x_{1}} p_{12}+\frac{p_{22}}{x_{1}} \frac{\partial v_{2}}{\partial x_{2}}\right) \\
& \frac{\delta p}{\delta t_{83}}=v_{1} \frac{\partial p_{83}}{\partial x_{1}}+\frac{v_{2}}{x_{1}} \frac{\partial p_{83}}{\partial x_{2}}-2\left\{\frac{\sin x_{2}}{h}\left(v_{1} p_{33}-v_{3} p_{18}\right)\right. \\
& \left.+\frac{\cos x_{2}}{h}\left(v_{2} p_{38}-v_{3} p_{28}\right)+p_{18} \frac{\partial v_{3}}{\partial x_{1}}+\frac{p_{28}}{x_{1}} \frac{\partial v_{8}}{\partial x_{2}}\right\} \\
& \frac{\delta p}{\delta t_{12}}=v_{1} \frac{\partial p_{12}}{\partial x_{1}}+\frac{v_{2}}{x_{1}} \frac{\partial p_{12}}{\partial x_{2}}-\frac{v_{1}}{x_{1}} p_{12} \\
& +\frac{v_{2}}{x_{1}} p_{11}-p_{12} \frac{\partial v_{1}}{\partial x_{1}}-\frac{p_{22}}{x_{1}} \frac{\partial v_{1}}{\partial x_{2}}-p_{11} \frac{\partial v_{2}}{\partial x_{1}}-\frac{p_{12}}{x_{1}} \frac{\partial v_{2}}{\partial x_{2}} \\
& \frac{\delta p}{\delta t_{18}}=v_{1} \frac{\partial p_{18}}{\partial x_{1}}+\frac{v_{2}}{x_{1}} \frac{\partial p_{18}}{\partial x_{2}}+\frac{\sin x_{2}}{h}\left(v_{8} p_{11}-v_{1} p_{18}\right) \\
& +\frac{\cos x_{2}}{h}\left(v_{8} p_{12}-v_{2} p_{18}\right)-p_{13} \frac{\partial v_{1}}{\partial x_{1}}-\frac{p_{28}}{x_{1}} \frac{\partial v_{1}}{\partial x_{2}} \\
& -p_{11} \frac{\partial v_{8}}{\partial x_{1}}-\frac{p_{12}}{x_{1}} \frac{\partial v_{8}}{\partial x_{2}} \\
& \frac{\delta p}{\delta t_{23}}=v_{1} \frac{\partial p_{23}}{\partial x_{1}}+\frac{v_{2}}{x_{1}} \frac{\partial p_{28}}{\partial x_{2}}-\frac{v_{1}}{x_{1}} p_{23}+\frac{v_{2}}{x_{1}} p_{13} \\
& -\frac{\sin x_{2}}{h}\left(v_{1} p_{23}-v_{3} p_{12}\right)-\frac{\cos x_{2}}{h}\left(v_{2} p_{23}-v_{3} p_{22}\right) \\
& -p_{18} \frac{\partial v_{2}}{\partial x_{1}}-\frac{p_{28}}{x_{1}} \frac{\partial v_{2}}{\partial x_{2}}-p_{12} \frac{\partial v_{8}}{\partial x_{1}}-\frac{p_{22}}{x_{1}} \frac{\partial v_{8}}{\partial x_{2}}
\end{aligned}
$$

\section{Equations for Calculation}

\subsection{Series expansion}

To solve eqs. (10)-(15), all variables are expanded into a series by powers of radius-ratio $1 / R$ as follows:

$$
\left.\begin{array}{l}
v_{i}=v_{i}(0)+\frac{1}{R} v_{i}^{(1)}+\frac{1}{R^{\mathbf{2}}} v_{i}^{(2)}+\cdots \\
p=p^{(0)}+\frac{1}{R} p^{(1)}+\frac{1}{R^{\mathbf{2}}} p^{(\mathbf{2})}+\cdots \\
p_{i j}=p_{i j}{ }^{(0)}+\frac{1}{R} p_{i j}^{(1)}+\frac{1}{R^{\mathbf{2}}} p_{i j}^{(2)}+\cdots \\
e_{i j}=e_{i j}^{(0)}+\frac{1}{R} e_{i j}^{(1)}+\frac{1}{R^{2}} e_{i j}^{(2)}+\cdots \\
\frac{\delta p}{\delta t_{i j}}=\frac{\delta p^{(0)}}{\delta t_{i j}}+\frac{1}{R} \frac{\delta p^{(1)}}{\delta t_{i j}}+\frac{1}{R^{\mathbf{2}}} \frac{\delta p^{(2)}}{\delta t_{i j}}+\cdots \\
M=M^{(0)}+\frac{1}{R} M^{(1)}+\frac{1}{R^{\mathbf{2}}} M^{(2)}+\cdots \\
\frac{1}{h}=\frac{1}{R}-\frac{1}{R^{\mathbf{2}}} x_{1} \sin x_{\mathbf{2}}+\frac{1}{R^{\mathbf{8}}} x_{1}{ }^{2} \sin ^{2} x_{2}+\cdots
\end{array}\right)
$$


Substitution of eq. (16) into eqs. (10), (11), (13), (15) and comparison of coefficients of the equal power of $1 / R$, yield equations for $v_{i}^{(m)}, p^{(m)}(m=0,1,2, \cdots)$.

\subsection{Flow in a straight pipe}

The zeroth approximate solution is obtained from zeroth coefficient equations. It represents the flow in a straight pipe $(R \rightarrow \infty)$ and is given by

$$
\begin{aligned}
& v_{1}{ }^{(0)}=v_{2}(0)=0, v_{8}(0)=1-x^{\frac{n+1}{n}} \\
& p^{(0)}=-\frac{2}{R_{e}}-\left(\frac{n+1}{n}\right)^{n} R x_{3} \\
& e_{13}^{(0)}=-\frac{1}{2} \frac{n+1}{n} x_{1}^{\frac{1}{n}}, e_{i j}^{(0)}=0\left(\text { except for } e_{13}^{(0)}\right) \\
& M^{(0)}=\left(\frac{n+1}{n}\right)^{2} x_{1}^{\frac{2}{n}} \\
& \frac{\delta p^{(0)}}{\delta t_{33}}=-\frac{2}{R_{\mathbf{c}}}\left(\frac{n+1}{n}\right)^{n+1} x_{1}{ }^{n+1} \\
& \left.\frac{\delta p^{(0)}}{\delta t_{i j}}=0 \text { (except for } \frac{\delta p^{(0)}}{\delta t_{33}}\right) \\
& p_{13}{ }^{(0)}=-\frac{1}{R_{e}}\left(\frac{n+1}{n}\right)^{n} x_{1}, \quad p_{33}{ }^{(0)}=\frac{W_{e}}{R_{e}}\left(\frac{n+1}{n}\right)^{s} x_{1}^{s} \\
& \left.p_{i j}{ }^{(0)}=0 \text { (except for } p_{13}{ }^{(0)}, p_{33}{ }^{(0)}\right)
\end{aligned}
$$

where $R x_{3}$ represents the length of pipe axis, and it is assumed that $p^{(0)}=0$ at $x_{3}=0$.

\subsection{First approximate solution}

Cauchy's equation of motion, the equation of continuity and the constitutive equation for the first approximate solution are from eq. (17)

$$
\begin{aligned}
& -v_{3}^{(0) 2} \sin x_{2}=-\frac{\partial p^{(1)}}{\partial x_{1}}+\frac{\partial p_{11}^{(1)}}{\partial x_{1}}+\frac{p_{11}{ }^{(1)}}{x_{1}} \\
& +\frac{1}{x_{1}} \frac{\partial p_{12}{ }^{(1)}}{\partial x_{2}}-\frac{p_{22}{ }^{(1)}}{x_{1}}-p_{33^{(0)}} \sin x_{2} \\
& -v_{3}{ }^{(0) 2} \cos x_{2}=-\frac{1}{x_{1}} \frac{\partial p^{(1)}}{\partial x_{2}}+\frac{\partial p_{12}{ }^{(1)}}{\partial x_{1}}+2 \frac{p_{12}{ }^{(1)}}{x_{1}} \\
& +\frac{1}{x_{1}} \frac{\partial p_{22}{ }^{(1)}}{\partial x_{2}}-p_{83}{ }^{(0)} \cos x_{2} \\
& v_{1}{ }^{(1)} \frac{\partial v_{8}{ }^{(0)}}{\partial x_{1}}=-\frac{1}{R} \frac{\partial p^{(1)}}{\partial x_{3}}+\frac{\partial p_{13}^{(1)}}{\partial x_{1}}+\frac{p_{13}{ }^{(1)}}{x_{1}} \\
& +\frac{1}{x_{1}} \frac{\partial p_{23}^{(1)}}{\partial x_{2}}+\frac{x_{1} \sin x_{2} \frac{\partial p^{(0)}}{\partial}}{R}+2 p_{13}^{(0)} \sin x_{2}
\end{aligned}
$$

$$
\begin{aligned}
& \frac{\partial v_{1}^{(1)}}{\partial x_{1}}+\frac{v_{1}^{(1)}}{x_{1}}+\frac{1}{x_{1}} \frac{\partial v_{2}^{(1)}}{\partial x_{2}}=0 \cdots \cdots \cdots \cdots \cdots \cdots \cdots(19) \\
& p_{11^{(1)}}=\frac{2}{R_{e}} M^{(0)^{n-1}=} e_{11}^{(1)}, p_{22}^{(1)}=\frac{2}{R_{e}} M^{(0)^{n-1}=} e_{22}^{(1)} \\
& p_{12}^{(1)}=\frac{2}{R_{e}} M^{(0)^{\frac{n-1}{2}}} e_{12}^{(1)} \\
& p_{13}^{(1)}=\frac{2}{R_{e}} M^{(0)^{\frac{n-1}{2}}}\left(\frac{n-1}{2} M^{(1)} M^{(0)} e_{13}^{(0)}+e_{13}^{(1)}\right) \\
& -W_{e} M^{(0)^{\frac{s-n-1}{2}}} \frac{\delta p^{(1)}}{\delta t_{13}}
\end{aligned}
$$

$$
\begin{aligned}
& p_{23}{ }^{(1)}=\frac{2}{R_{e}} M^{(0)^{\frac{n-1}{2}}} e_{23}^{(1)}-W_{e} M^{(0) \frac{s-n-1}{2}} \frac{\delta p^{(1)}}{\delta t_{28}} \\
& p_{33}{ }^{(1)}=-W_{e} M^{(0)^{\frac{s-n-1}{2}}}\left(\frac{s-n-1}{2} \frac{M^{(1)}}{M^{(0)}} \frac{\delta p^{(0)}}{\delta t_{33}}+\frac{\delta p^{(1)}}{\delta t_{83}}\right)
\end{aligned}
$$

where

$$
\begin{aligned}
& e_{11}^{(1)}=\frac{\partial v_{1}^{(1)}}{\partial x_{1}}, \quad e_{22}^{(1)}=\frac{1}{x_{1}} \frac{\partial v_{2}^{(1)}}{\partial x_{2}}+\frac{v_{1}^{(1)}}{x_{1}} \\
& e_{33^{(1)}}=0, \quad e_{12}^{(1)}=\frac{1}{2}\left(\frac{1}{x_{1}} \frac{\partial v_{1}^{(1)}}{\partial x_{2}}+\frac{\partial v_{2}^{(1)}}{\partial x_{1}}-\frac{v_{2}^{(1)}}{x_{1}}\right) \\
& e_{13}^{(1)}=\frac{1}{2}\left(\frac{\partial v_{3}{ }^{(1)}}{\partial x_{1}}-v_{3}{ }^{(0)} \sin x_{2}\right) \\
& e_{23}{ }^{(1)}=\frac{1}{2}\left(\frac{1 \partial v_{3}^{(1)}}{x_{1} \partial x_{2}}-v_{3}{ }^{(0)} \cos x_{2}\right) \\
& M^{(1)}=8 e_{13}^{(0)} e_{13}^{(1)} \\
& \frac{\delta p^{(1)}}{\delta t_{11}}=\frac{\delta p^{(1)}}{\delta t_{22}}=\frac{\delta p^{(1)}}{\delta t_{12}}=0 \\
& \frac{\delta p^{(1)}}{\delta t_{13}}=v_{(1)} \frac{\partial p_{18}{ }^{(0)}}{\partial x_{1}}-p_{13}{ }^{(0)} \frac{\partial v_{1}^{(1)}}{\partial x_{1}}-p_{11}{ }^{(1)} \frac{\partial v_{3}{ }^{(0)}}{\partial x_{1}}
\end{aligned}
$$

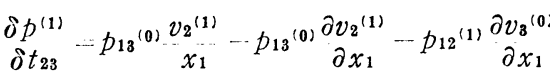

$$
\begin{aligned}
& \frac{\delta p^{(1)}}{\delta t_{33}}=v_{1}{ }^{(1)} \frac{\partial p_{33}(0)}{\partial x_{1}} \\
& \left.-2\left(p_{13}{ }^{(0)} \frac{\partial v_{3}^{(1)}}{\partial x_{1}}+p_{13}{ }^{(1)} \frac{\partial v_{3}^{(0)}}{\partial x_{1}}-v_{3}^{(0)} p_{13}^{(0)} \sin x_{2}\right)\right)
\end{aligned}
$$

The boundary condition is given by

$$
\left.\begin{array}{l}
v_{i}^{(1)}=0 \quad \text { on the pipe wall } x_{1}=1 . \\
v_{i}^{(1)}=(\text { finite }) \quad \text { inside the pipe } 0 \leqq x_{1}<1 \\
p^{(1)}=0 \quad \text { at the pipe center } x_{1}=0
\end{array}\right\}
$$

The solution of eqs. (18)-(22) can be represented by

$$
\begin{aligned}
& v_{1}{ }^{(1)}=V_{1}\left(x_{1}\right) \sin x_{2} \\
& v_{2}^{(1)}=V_{2}\left(x_{1}\right) \cos x_{2} \\
& v_{3}{ }^{(1)}=V_{3}\left(x_{1}\right) \sin x_{2} \\
& p^{(1)}=P\left(x_{1}\right) \sin x_{2}
\end{aligned}
$$

Substituting eq. (23) into eqs. (18)-(22), eliminating $P$ from the first and the second equations of eq. (18) and eliminating $V_{2}$ by the use of eq. (19) yield the following differential equation for $V_{1}\left(x_{1}\right)$ :

$$
\begin{aligned}
& x_{1}{ }^{4} V_{1^{\prime \prime \prime \prime \prime}}+\frac{8 n-2}{n} x_{1^{3}} V_{1^{\prime \prime \prime}}+\frac{10 n^{2}-8 n+1}{n^{2}} x_{1}{ }^{2} V_{1}{ }^{\prime \prime}
\end{aligned}
$$

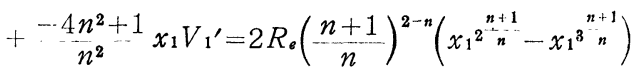

$$
\begin{aligned}
& +W \cdot\left(\frac{n+1}{n}\right)^{s-n+1} \frac{s}{n} x_{1}^{s+n+1}
\end{aligned}
$$


where the prime denotes differentiation with respect to $x_{1}$. The general solution of eq. (24) in case that $n \neq 1$ is

$$
\begin{aligned}
& V_{1}=c_{1}+c_{2} x_{1}{ }^{1-n+\sqrt{17 n^{2}-2 n+1}}+c_{4} x_{1} \frac{1-n-\sqrt{17 n^{2}-2 n+1}}{2 n} \\
& +c_{5} x_{1} \frac{1-n}{n}+R \cdot\left(\frac{n+1}{n}\right)^{1-n} \frac{n^{3}}{2(3 n+1)\left(n^{2}+4 n+1\right)} x_{1^{2}}{ }^{\frac{n+1}{n}} \\
& -R \cdot\left(\frac{n+1}{n}\right)^{1-n} \frac{n^{3}}{6(2 n+1)\left(4 n^{2}+9 n+3\right)} x^{x^{3}{ }^{n+1}} \\
& +W_{e} \cdot\left(\frac{n+1}{n}\right)^{s-n+1} \\
& \times \frac{s n^{3}}{(s+n+1)(s+2 n)\left(s^{2}+3 s n+s-2 n^{2}+2 n\right)} x^{x_{1}^{s+n+1}}
\end{aligned}
$$

where $c_{1}, c_{2}, c_{4}$ and $c_{5}$ are the integration constants. In case that $n=1, c_{5} x_{1}(1-n) / n$ in eq. (25) is replaced by $c_{5} \ln x_{1}$. After $V_{1}$ is obtained, $V_{2}$ becomes from the equation of continuity (19)

$$
V_{2}=x_{1} V_{1}^{\prime}+V_{1}
$$

The constants $c_{4}$ and $c_{5}$ are

$$
c_{4}=c_{5}=0
$$

because the physical values must be finite at the pipe center $x_{1}=0$. The constants $c_{1}$ and $c_{2}$ can be obtained for the flow velocities to vanish on the pipe wall.

On substituting the above results into the third equation of eq. (18), the equation for $V_{3}\left(x_{1}\right)$ is given by

$$
\begin{aligned}
& x_{1}{ }^{2} V_{3}{ }^{\prime \prime}+\frac{2 n-1}{n} x_{1} V_{3^{\prime}}-\frac{1}{n} V_{3}=2 \frac{n-1}{n} x_{1} \\
& +\frac{-3 n^{2}+5 n+4}{n^{2}} x_{1}{ }^{2 n+1} \\
& -R_{e}\left(\frac{n+1}{n}\right)^{2-n} \frac{c_{1}}{n} x_{1}^{\frac{n+2}{n}} \\
& -R_{e}\left(\frac{n+1}{n}\right)^{2-n} \frac{c_{2}}{n} x_{1} \frac{n+5+\sqrt{17 n^{2}-2 n+1}}{2 n} \\
& -R_{e}^{2}\left(\frac{n+1}{n}\right)^{3-2 n} \frac{n^{2}}{2(3 n+1)\left(n^{2}+4 n+1\right)} x_{1}^{\frac{3 n+4}{n}} \\
& +R_{e}^{2}\left(\frac{n+1}{n}\right)^{3-2 n} \frac{n^{2}}{6(2 n+1)\left(4 n^{2}+9 n+3\right)} x_{1}^{\frac{4 n+5}{n}} \\
& -W_{\cdot}\left(\frac{n+1}{n}\right)^{s-n} \frac{s-n-1}{n^{2}} c_{1} x_{1}{ }^{s-n} \\
& +W_{e}\left(\frac{n+1}{n}\right)^{s-n}\left\{12 n^{2}+2 n-2-5 s n+3 s+(3 s\right. \\
& \left.-2 n-2) \sqrt{17 n^{2}-2 n+1}\right\} \frac{c_{2}}{2 n^{3}} x_{1} \frac{2 s-3 n+1+\sqrt{17 n^{2}-2 n+1}}{2 n} \\
& +W_{e} R_{e}\left(\begin{array}{c}
n+1 \\
n
\end{array}\right)^{s-2 n+1} \frac{3 n^{2}+n-2+5 s n+6 s}{2(3 n+1)\left(n^{2}+4 n+1\right)} x_{1}^{\frac{s+n+2}{n}} \\
& -W_{e} R_{e}\left(\frac{n+1}{n}\right)^{s-2 n+1} \\
& \times\left\{\frac{s(n+1)^{2}}{(s+n+1)(s+2 n)\left(s^{2}+3 s n+s-2 n^{2}+2 n\right)}\right.
\end{aligned}
$$

$$
\begin{aligned}
& \left.+\frac{7 n^{2}+7 n+8 s n+9 s}{6(2 n+1)\left(4 n^{2}+9 n+3\right)}\right\} x_{1}^{\frac{s+2 n+8}{n}} \\
& +W_{e}^{2}\left(\frac{n+1}{n}\right)^{2 s-2 n+1} \\
& \times \frac{s\left(4 s^{2}+3 s n+2 s+n^{2}-n-2\right)}{(s+n+1)(s+2 n)\left(s^{2}+3 s n+s-2 n^{2}+2 n\right)} x_{1}^{\frac{2 s+1}{n}}
\end{aligned}
$$

The general solution of eq. (28) is

$$
\begin{aligned}
& V_{3}=c_{8} x_{1}^{\frac{1}{n}}+x_{1}+\frac{-3 n^{2}+5 n+4}{2 n(3 n+1)} x_{1}{ }^{\frac{2 n+1}{n}} \\
& -R \cdot\left(\frac{n+1}{n}\right)^{-n} \frac{c_{1}}{2 n} x_{1}{ }^{\frac{n+2}{n}} \\
& -R \cdot\left(\frac{n+1}{n}\right)^{2-n} \frac{n c_{2}}{5 n^{2}+3 n+4+(n+2) \sqrt{17 n^{2}-2 n+1}} \\
& \times x_{1}^{\frac{n+5+\sqrt{17 n^{2}-2 n+1}}{2 n}} \\
& -R_{e}^{2}\left(\frac{n+1}{n}\right)^{1-2 n} \frac{n^{2}}{24(3 n+1)\left(n^{2}+4 n+1\right)} x_{1}^{\frac{3 n+4}{n}} \\
& +\operatorname{Re}^{2}\left(\frac{n+1}{n}\right)^{1-2 n} \frac{n^{2}}{120(2 n+1)\left(4 n^{2}+9 n+3\right)} x_{1}^{\frac{4 n+5}{n}} \\
& -W_{e}\left(\frac{n+1}{n}\right)^{s-n} \frac{c_{1}}{s} x_{1}{ }^{s-n}-W_{e}\left(\frac{n+1}{n}\right)^{s-n} \\
& \times\left\{-12 n^{2}-2 n+2+5 s n-3 s+(-3 s+2 n+2)\right. \\
& \left.\times \sqrt{17 n^{2}-2 n+1}\right\} /\left\{10 n^{2}-2 n+2 s^{2}-4 s n+2(s-n)\right. \\
& \left.\times \sqrt{17 n^{2}-2 n+1}\right\} \frac{c_{2}}{n} x_{1} \frac{2 s-3 n+1+\sqrt{17 n^{2}-2 n+1}}{2 n} \\
& +W_{e} R_{e}\left(\frac{n+1}{n}\right)^{s-2 n+1} \frac{n^{2}\left(3 n^{2}+n-2+5 s n+6 s\right)}{2(3 n+1)(s+n+1)} \\
& \times(s+2 n+2)\left(n^{2}+4 n+1\right)^{x_{1}^{s+n+2}} \\
& -W_{e} R_{e}\left(\frac{n+1}{n}\right)^{s-2 n+1}\left\{\begin{array}{c}
s n^{2}(n+1)^{2} \\
(s+n+1)(s+2 n+2)
\end{array}\right. \\
& \times \frac{1}{(s+3 n+3)(s+2 n)\left(s^{2}+3 s n+s-2 n^{2}+2 n\right)} \\
& +n^{2}\left(7 n^{2}+7 n+8 s n+9 s\right) \\
& 6(2 n+1)\left(4 n^{2}+9 n+3\right) \\
& \left.\times \frac{1}{s^{2}+5 s n+5 s+6 n^{2}+12 n+6}\right\} x_{1}^{\frac{s+2 n+3}{n}} \\
& +W_{e}^{2}\left(\frac{n+1}{n}\right)^{2 s-2 n+1} \frac{n^{2}\left(4 s^{2}+3 s n+2 s+n^{2}-n-2\right)}{2(s+n+1)(s+2 n)(2 s+n+1)} \\
& \times \frac{1}{s^{2}+3 s n+s-2 n^{2}+2 n} x_{1}^{\frac{2 s+1}{n}}+c_{6} x_{1}^{-1}
\end{aligned}
$$

where $c_{3}$ and $c_{6}$ are the integration constants. Because $v_{3}$ is finite at $x_{1}=0, c_{6}$ is

$$
c_{6}=0
$$

The constant $c_{3}$ is determined for $V_{3}$ to vanish on the pipe wall.

3.4 Flow velocity, isotropic pressure, deviatric stress and stream line 
From the results obtained hereunto, the flow velocity and the isotropic pressure are as follows:

$$
\begin{aligned}
v_{1} & =\frac{1}{R} V_{1}\left(x_{1}\right) \sin x_{2} \\
& =\frac{1}{R}\left[c_{1}+c_{2} x_{1} \frac{1-n+\sqrt{17 n^{2}-2 n+1}}{2 n}\right. \\
& +R_{\bullet}\left(\frac{n+1}{n}\right)^{1-n} \frac{n^{3}}{2(3 n+1)\left(n^{2}+4 n+1\right)} x_{1^{2}}^{2^{n+1}} \\
& -R_{\bullet}\left(\frac{n+1}{n}\right)^{1-n} \frac{n^{3}}{6(2 n+1)\left(4 n^{2}+9 n+3\right)} x_{1^{3}}^{3^{\frac{n+1}{n}}} \\
& +W_{\bullet}\left(\frac{n+1}{n}\right)^{s-n+1} \frac{s n^{3}}{(s+n+1)(s+2 n)} \\
& \left.\times \frac{1}{s^{2}+3 s n+s-2 n^{2}+2 n} x^{\frac{s+n+1}{n}}\right] \sin x_{2} \cdots \cdots
\end{aligned}
$$

$v_{2}=\frac{1}{R} V_{2}\left(x_{1}\right) \cos x_{2}$

$=\frac{1}{R}\left[c_{1}+\frac{n+1+\sqrt{17 n^{2}-2 n+1}}{2 n} c_{2} x_{1} \frac{1-n+\sqrt{17 n^{2}-2 n+1}}{2 n}\right.$

$+R_{\bullet}\left(\frac{n+1}{n}\right)^{1-n} \frac{n^{2}(3 n+2)}{2(3 n+1)\left(n^{2}+4 n+1\right)} x_{1^{2}}{ }^{\frac{n+1}{n}}$

$-R_{e}\left(\frac{n+1}{n}\right)^{1-n} \frac{n^{2}(4 n+3)}{6(2 n+1)\left(4 n^{2}+9 n+3\right)} x_{1^{3^{\frac{n+1}{n}}}}$

$+W_{e}\left(\frac{n+1}{n}\right)^{s-n+1} \frac{s n^{2}(s+2 n+1)}{(s+n+1)(s+2 n)}$

$\left.\times \frac{1}{s^{2}+3 s n+s-2 n^{2}+2 n} x_{1}{ }^{\frac{s+n+1}{n}}\right] \cos x_{2}$

$v_{3}=1-x_{1}{ }^{n+1}{ }^{n}+\frac{1}{R} V_{3}\left(x_{1}\right) \sin x_{2}$

$-1-x_{1}{ }^{\frac{n+1}{n}}+\frac{1}{R}\left[c_{3} x_{1}^{\frac{1}{n}}+x_{1}+\frac{-3 n^{2}+5 n+4}{2 n(3 n+1)} x_{1}{ }^{\frac{2 n+1}{n}}\right.$

$-R_{e}\left(\frac{n+1}{n}\right)^{-n} \frac{c_{1}}{2 n} x_{1}{ }^{n+2}$

$-R_{e}\left(\frac{n+1}{n}\right)^{2-n} \frac{n c_{2}}{5 n^{2}+3 n+4+(n+2) \sqrt{17 n^{2}}-2 n+1}$

$$
\times x_{1} \frac{n+5+\sqrt{17 n^{2}-2 n+1}}{2 n}
$$

$-R_{e}^{2}\left(\frac{n+1}{n}\right)^{1-2 n} \frac{n^{2}}{24(3 n+1)\left(n^{2}+4 n+1\right)} x_{1}{ }^{\frac{3 n+4}{n}}$

$+R_{e}{ }^{2}\left(\frac{n+1}{n}\right)^{1-2 n} \frac{n^{2}}{120(2 n+1)\left(4 n^{2}+9 n+3\right)} x_{1}{ }^{4 n+5}$

$-W \cdot\left(\frac{n+1}{n}\right)^{s-n} \frac{c_{1}}{s} x_{1} \frac{s-n}{n}-W \cdot\left(\frac{n+1}{n}\right)^{s-n}$

$\times\left\{-12 n^{2}-2 n+2+5 s n-3 s+(-3 s+2 n+2)\right.$

$\left.\times \sqrt{17 n^{2}-2 n+1}\right\} /\left\{10 n^{2}-2 n+2 s^{2}-4 s n+2(s-n)\right.$

$\left.\times \sqrt{17 n^{2}-2 n+1}\right\} \frac{C_{2}}{n} x_{1} \frac{2 s-3 n+1+\sqrt{17 n^{2}-2 n+1}}{2 n}$

$+W \cdot R \cdot\left(\frac{n+1}{n}\right)^{s-2 n+1} \frac{n^{2}\left(3 n^{2}+n-2+5 s n+6 s\right)}{2(3 n+1)(s+n+1)}$

$$
\begin{aligned}
& \times \frac{1}{(s+2 n+2)\left(n^{2}+4 n+1\right)} x_{1}^{\frac{s+n+2}{n}} \\
& -W_{e} R_{e}\left(\frac{n+1}{n}\right)^{s-2 n+1}\left\{\frac{s n^{2}(n+1)^{2}}{(s+n+1)(s+2 n+2)}\right. \\
& \times \frac{1}{(s+3 n+3)(s+2 n)\left(s^{2}+3 s n+s-2 n^{2}+2 n\right)} \\
& +\frac{n^{2}\left(7 n^{2}+7 n+8 s n+9 s\right)}{6(2 n+1)\left(4 n^{2}+9 n+3\right)} \\
& \left.\times \frac{1}{s^{2}+5 s n+5 s+6 n^{2}+12 n+6}\right\} x_{1}^{\frac{s+2 n+3}{n}} \\
& +W \cdot \mathbf{c}^{\prime}\left(\frac{n+1}{n}\right)^{2 s-2 n+1} \frac{n^{2}\left(4 s^{2}+3 s n+2 s+n^{2}-n-2\right)}{2(s+n+1)(s+2 n)(2 s+n+1)} \\
& \left.\times \frac{1}{s^{2}+3 s n+s-2 n^{2}+2 n} x_{1}^{\frac{2 s+1}{n}}\right] \sin x_{2} \\
& p=-\frac{2}{R_{e}}\left(\frac{n+1}{n}\right)^{n} R x_{3}+\frac{1}{R} P\left(x_{1}\right) \sin x_{2} \\
& =-\frac{2}{R_{e}}\left(\frac{n+1}{n}\right)^{n} R x_{3} \\
& +\frac{1}{R}\left[\frac{1}{R_{6}}\left(\frac{n+1}{n}\right)^{n-1} \frac{7 n^{2}+1+(n+1) \sqrt{17 n^{2}-2 n+1}}{2 n^{2}}\right. \\
& \times c_{2} x_{1} \frac{\frac{-n-1+\sqrt{17 n^{2}-2 n+1}}{2 n}}{2 n}+x_{1}-\frac{2 n(5 n+1)}{(3 n+1)\left(n^{2}+4 n+1\right)} \\
& \times x_{1} \frac{2 n+1}{n}+\frac{n(3 n+1)(n+3)}{2(2 n+1)\left(4 n^{2}+9 n+3\right)} x_{1}^{\frac{3 n+2}{n}} \\
& -\frac{W_{e}}{R_{e}}\left(\frac{n+1}{n}\right)^{s} \frac{n(s+4 n)(s-n+1)}{(s+2 n)\left(s^{2}+3 s n+s-2 n^{2}+2 n\right)} \\
& \left.\times x_{1} \frac{s+n}{n}\right] \sin x_{2}
\end{aligned}
$$

where the constants $c_{1}, c_{2}$ and $c_{3}$ are

$$
\begin{aligned}
c_{1} & =R_{e}\left(\frac{n+1}{n}\right)^{1-n} n\left\{3(13 n+5)(n+1)^{3} \sqrt{ } 17 n^{2}-2 n+1\right. \\
& \left.-129 n^{5}-331 n^{4}-274 n^{3}-142 n^{2}-69 n-15\right\} \\
& /\left\{48(2 n+1)(3 n+1)\left(n^{2}+4 n+1\right)\left(4 n^{2}+9 n+3\right)\right\} \\
& +W_{e}\left(\frac{n+1}{n}\right)^{s-n+1} s n\left\{(s+n+1) \sqrt{17 n^{2}-2 n+1}\right. \\
& \left.+s n-s-7 n^{2}-1\right\} /\left\{8 ( s + n + 1 ) ( s + 2 n ) \left(s^{2}+3 s n\right.\right. \\
& \left.\left.+s-2 n^{2}+2 n\right)\right\} \\
c_{2} & =\left(1-n-\sqrt{\left.17 n^{2}-2 n+1\right)}\left[R_{e}\left(\frac{n+1}{n}\right)^{4-n}\right.\right. \\
& \times-16(2 n+1)(3 n+1)\left(n^{2}+4 n+1\right)\left(4 n^{2}+9 n+3\right) \\
& +W_{e}\left(\frac{n+1}{n}\right)^{s-n+1} \\
& \times-8(s+2 n)\left(s^{2}+3 s n+s-2 n^{2}+n\right) \\
c_{3} & =-\frac{3 n^{2}+7 n+4}{2 n(3 n+1)}+R_{e}\left(\frac{n+1}{n}\right)^{-n} \frac{1}{n}\left\{\frac{c_{1}}{2}\right. \\
& \left.+\frac{(n+1)^{2} c_{2}}{5 n^{2}+3 n+4+(n+2) \sqrt{17 n^{2}-2 n+1}}\right\} \\
& +R_{e}^{2}\left(\frac{n+1}{n}\right)^{1-2 n}\left\{\frac{n^{2}}{24(3 n+1)\left(n^{2}+4 n+1\right)}\right. \\
&
\end{aligned}
$$




$$
\begin{aligned}
& \left.-\frac{n^{2}}{120(2 n+1)\left(4 n^{2}+9 n+3\right)}\right\} \\
& +W \cdot\left(\frac{n+1}{n}\right)^{s-n}\left[\frac{c_{1}}{s}+\left\{-12 n^{2}-2 n+2+5 s n-3 s\right.\right. \\
& \left.+(-3 s+2 n+2) \sqrt{17 n^{2}-2 n+1}\right\} /\left\{10 n^{2}-2 n+2 s^{2}\right. \\
& \left.\left.-4 s n+2(s-n) \sqrt{17 n^{2}-2 n+1}\right\} \frac{c_{2}}{n}\right] \\
& -W \cdot R \cdot\left(\frac{n+1}{n}\right)^{s-2 n+1} \\
& \times\left\{\frac{n^{2}\left(3 n^{2}+n-2+5 s n+6 s\right)}{2(3 n+1)(s+n+1)(s+2 n+2)\left(n^{2}+4 n+1\right)}\right. \\
& -\frac{s n^{2}(n+1)^{2}}{(s+n+1)(s+2 n+2)(s+3 n+3)(s+2 n)} \\
& \times \frac{1}{s^{2}+3 s n+s-2 n^{2}+2 n}-\frac{n^{2}}{6(2 n+1)} \\
& \left.\times \frac{7 n^{2}+7 n+8 s n+9 s}{\left(4 n^{2}+9 n+3\right)\left(s^{2}+5 s n+5 s+6 n^{2}+12 n+6\right)}\right\} \\
& -W e^{2}\left(\frac{n+1}{n}\right)^{2 s-2 n-1} n^{2}\left(4 s^{2}+3 s n+2 s+n^{2}-n-2\right) \\
& 2(s+n+1)(s+2 n) \\
& \times \frac{1}{(2 s+n+1)\left(s^{2}+3 s n+s-2 n^{2}+2 n\right)} \\
&
\end{aligned}
$$

The deviatric stress is as follows:

$$
\begin{aligned}
p_{11} & =\frac{1}{R} \frac{2}{R_{e}}\left(\frac{n+1}{n}\right)^{n-1} x_{1}^{\frac{n-1}{n}} V_{1^{\prime}} \sin x_{2} \\
p_{22} & =-\frac{1}{R} \frac{2}{R_{e}}\left(\frac{n+1}{n}\right)^{n-1} x_{1}^{\frac{n-1}{n}} V_{1^{\prime}} \sin x_{2} \\
p_{12} & =\frac{1}{R} \frac{1}{R_{e}}\left(\frac{n+1}{n}\right)^{n-1} x_{1}^{\frac{n-1}{n}}\left(V_{2^{\prime}}-V_{1^{\prime}}\right) \cos x_{2} \\
p_{13} & =-\frac{1}{R_{e}}\left(\frac{n+1}{n}\right)^{n} x_{1} \\
& +\frac{1}{R}\left[\frac{n}{R_{e}}\left(\frac{n+1}{n}\right)^{n-1} x_{1}{ }^{\frac{n-1}{n}}\left(V_{3^{\prime}}-1+x_{1} \frac{n+1}{n}\right)\right. \\
& \left.+\frac{W_{e}}{R_{e}}\left(\frac{n+1}{n}\right)^{s-1} x_{1}^{\frac{s-n-1}{n}}\left(V_{1}-3 x_{1} V_{1^{\prime}}\right)\right] \sin x_{2} \\
p_{23} & =\frac{1}{R}\left[\frac{1}{R_{e}}\left(\frac{n+1}{n}\right)^{n-1} x_{1}{ }^{\frac{n-1}{n}}\left(\frac{V_{3}}{x_{1}}-1+x_{1}{ }^{\frac{n+1}{n}}\right)\right. \\
& \left.+\frac{W_{e}}{R_{e}}\left(\frac{n+1}{n}\right)^{s-1} x_{1}^{\frac{s-1}{n}}\left(-\frac{V_{1}}{x_{1}}+2 \frac{V_{2}}{x_{1}}-2 V_{2^{\prime}}\right)\right] \cos x_{2} \\
p_{33} & =\frac{W_{e}}{R_{e}}\left(\frac{n+1}{n}\right)^{s} x_{1}^{\frac{s}{n}} \\
& +\frac{1}{R}\left[-2 \frac{W_{e}}{R_{e}}\left(\frac{n+1}{n}\right)^{s-1} s x_{1}^{\frac{s-1}{n}}\left(V_{3^{\prime}}-1+x_{1}^{\frac{n+1}{n}}\right)\right. \\
& -\frac{W_{e}{ }^{2}}{R_{e}}\left(\frac{n+1}{n}\right)^{2 s-n-1} x_{1}^{\frac{2 s-2 n-1}{n}}\left(\frac{s+2 n^{\prime}}{n} V_{1}\right. \\
& \left.\left.-6 x_{1} V_{1^{\prime}}\right)\right] \sin x_{2}
\end{aligned}
$$

To determine the stream line of the secondary flow on the cross-sectional plane $x_{3}=$ (const.) of the pipe, the stream function $\phi$ is defined as

$$
\left.\begin{array}{l}
v_{1}=-\frac{1}{x_{1}} \frac{\partial \psi}{\partial x_{2}}, \quad v_{2}=\frac{\partial \psi}{\partial x_{1}} \\
\psi=0 \quad \text { at } x_{1}=1
\end{array}\right\} \cdots
$$

Then, the stream function is given by

$$
\psi=\frac{1}{R} x_{1} V_{1}\left(x_{1}\right) \cos x_{2}
$$

The stream line on the central plane $x_{2}= \pm \pi / 2$ is obtained by numerically integrating

$$
x_{3}=\frac{1}{\sin x_{2}} \int_{0}^{x_{1}} \frac{1-x_{1}^{\frac{n+1}{n}}}{V_{1}} d x_{1}
$$

because the equation for the stream line on $x_{2}= \pm \pi / 2$ is

$$
\frac{d x_{1}}{v_{1}}=\frac{h d x_{3}}{v_{3}}
$$

\section{Calculated Examples of Stream Lines and Axial Flow Velocity}

Eq. (38) for the stream function $\phi$ showing the stream line of the secondary flow on the cross-sectional plane $x_{3}=$ (const.) of the pipe can be rewritten as

$$
\psi=\frac{1}{R}\left[\psi_{a}\left(n, x_{1}\right) R_{e}+\psi_{b}\left(n, s, x_{1}\right) W_{e}\right] \cos x_{2} \cdots(41)
$$

The distributions of the coefficients $\psi_{a}$ and $\psi_{b}$ in eq. (41) for some viscosity index $n$ and elasticity index $s$ are shown in Fig. 2. Values of the stream function $\phi$ are illustrated in Fig. 3. Points $\mathbf{P}_{i}$ and $\mathbf{P}_{o}$ are intersections of line OC and the pipe wall, and points $\mathbf{P}_{u}$ and $\mathbf{P}_{l}$ are intersections of a line parallel to $z$ axis through the central point $C$ and the pipe wall as shown Fig. 1. The symbol $\times$ represents a central point of the spiral and the arrow shows a flow direction. The stream lines are drawn in quarter parts of a circle, because they are symmetric with respect to the lines $\mathbf{P}_{i} \mathbf{P}_{o}$ and $\mathbf{P}_{u} \mathbf{P}_{l}$ in case that the stream function is given by eq. (41) represented in the series to the first term. In the case of Power law fluid the maximum value of $\psi_{a}$ and the flow rate of the secondary flow are larger for smaller $n$ as shown in Figs. 2 and 3 (a), (b). The central points of the spiral move nearer to the pipe wall remarkablly for smaller $n$. In the case of White-Metzner fluid the value of $\psi_{b}$ is larger than that of $\psi_{a}$ as shown in Fig. 2, and the elastic force increases the flow rate of the secondary flow similarly to the earlier results $^{[4,6,7]}$. From Fig. 3(c), (d), the smaller $n$ is and the larger $s$ is, the larger $\phi_{b}$ is and the larger the degree of increase in the flow rate of the secondary flow. For fixed $n$, the radial position $x_{1}$ giving the maximum value of $\phi_{b}$ is somewhat larger than that of $\psi_{a}$, and the elastic force shifts the central position of the spiral to the pipe wall (Figs. 2 and 3 (b), (c), (d)). But the viscosity index $n$ is the maximum factor 


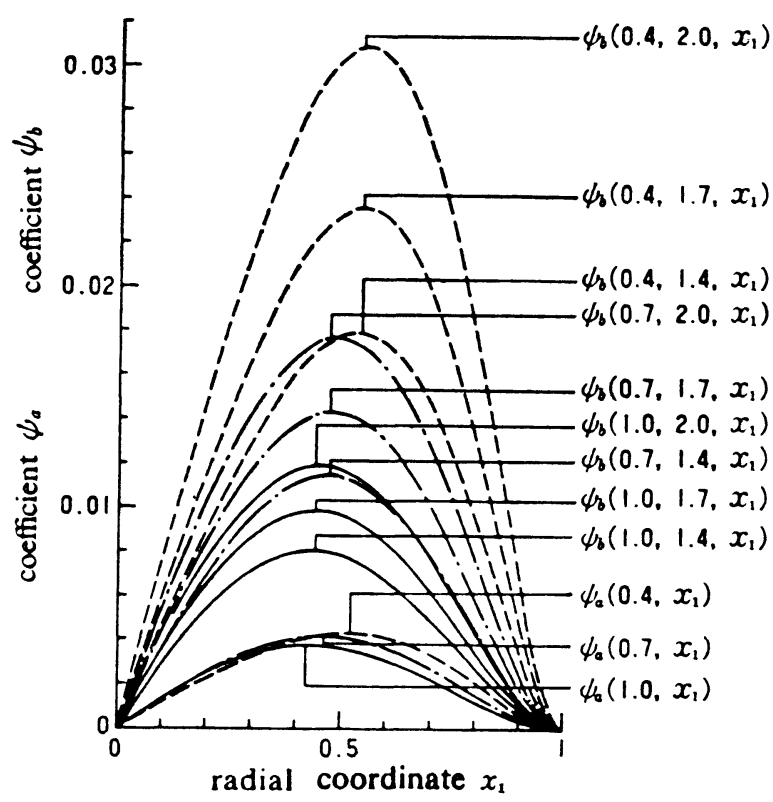

Fig. 2 Distributions of coefficients $\psi_{a}\left(n, x_{1}\right)$ and $\psi_{b}\left(n, s, x_{1}\right)$

effective to shift the central point toward the pipe wall. From eq. (41) the flow rate of the secondary flow increases for larger $R e$ and $W e$ because both $\psi_{a}$ and $\psi_{b}$ are plus.

Equation (33) for the axial flow velocity $v_{3}$ can be rewritten as

$$
\begin{aligned}
& v_{3} v_{3}{ }^{0}\left(n, x_{1}\right): \\
& { }_{R}\left[v_{a}\left(n, x_{1}\right)+v_{b}\left(n, x_{1}\right) R_{e}{ }^{2}\right. \\
& \left.+v_{c}\left(n, s, x_{1}\right) R_{e} W_{e}+v_{d}\left(n, s, x_{1}\right) W_{e}^{2}\right] \sin x_{2}
\end{aligned}
$$

Figure 4 shows the zeroth approximate solution $v_{3}{ }^{(0)}=1-$ $x_{1}{ }^{(n+1) / n}$ and the coefficients $v_{a}, v_{b}, v_{c}, v_{d}$ of the first approximate solution. Figure 5 shows the distributions of the axial flow velocity $v_{3}{ }^{(0)}$ in a straight pipe and the axial flow velocity $v_{3}$ on the central plane $\mathrm{P}_{i} \mathrm{P}_{o}$ for $R=3$. The distribution for Power law fluid is in Fig. 5 (a) and that for WhiteMetzner fluid being in Fig. 5(b). The symbol $\bigcirc$ shows the position where the axial flow velocity is the maximum. The axial flow velocity of Power law fluid is represented by $v_{3}{ }^{(0)}$, $v_{a}$ and $v_{b}$ alone. As well-known, $v_{3}{ }^{(0)}$ shows a gently-sloping distribition in the vicinity of the pipe center for small $n$ (Fig. 4(a)). The coefficient $v_{a}$ for $n=1$. shows a minus value Hence, in the case of small $R e$ the maximum axial flow velocity is near $z$ axis (near point $\mathrm{P}_{i}$ ) (Fig. $5(\mathrm{a})$ ). With the decrease of $n$ it moves nearer to point $\mathbf{P}_{o}$. As $v_{b}$ is always plus and the centrifugal force increases with the increment of $R e$, the position of the maximum axial flow velocity shifts toward point $\mathrm{P}_{0}$. The coefficients $v_{c}$ and $v_{d}$ in Fig. 4(b), (c) represent the influence of the elastic force, and their maximum values are large when $n$ is small and $s$ is large. The
(a) viscosity index
$n=1.0$
(b) $n=0.4 \quad R_{\theta}=1$
Reynolds number $\quad \mathrm{Re}=1$ elasticity index $\quad s=-$
Weissenberg number $W_{\theta}=0$
$s=-W_{e}=0$

(Newtonian fluid)

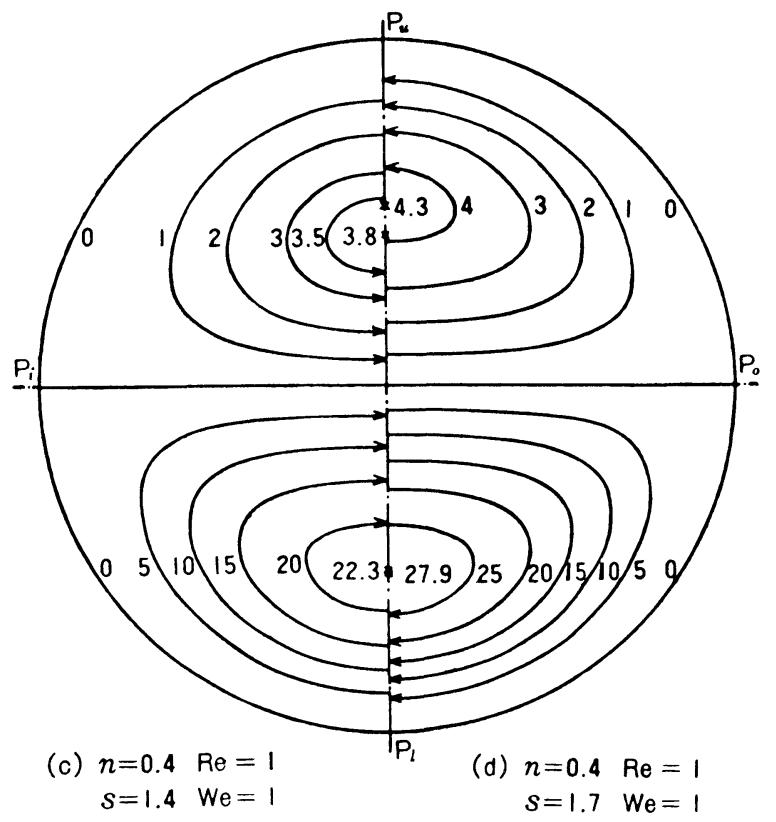

Fig. 3 Values of stream function showing secondary flow (values of $\phi R \times 10^{3}$ in Figs. (a), (b) and $-\psi R \times 10^{3}$ in Figs. (c), (d))

position exists where the values of $v_{c}$ and $v_{d}$ are minus for small $s$. The position of the maximum axial flow velocity (Fig. 5(b)) moves toward point $\mathrm{P}_{i}$ and that degree is larger for larger $W e$.

Fig. 6 shows the stream line on the central plane. The arrow there represents the flow direction. Eq. (39) for the stream line on the central plane does not contain $R$ denoting the reciprocal of the radius-ratio, but in Fig. 6 the stream line for $R=3$ is drawn. Eq. (39) can be rewritten as

$$
\begin{aligned}
x_{3}= & \frac{1}{} \\
& \sin x_{2} \\
& \cdots \int_{0}^{x_{1}}\left\{\psi_{a}\left(n, x_{1}\right) / x_{1}\right\} R_{e}+\left\{\psi_{b}^{(0)}\left(n, x_{1}\right)\right. \\
&
\end{aligned}
$$

As $\phi^{\prime} a / x_{1}$ and $\phi^{\prime} / x_{1}$ are plus, for larger $R e$ and $W e$ the outward shift of the stream line on the central plane starting at point $x_{1}=0$ and $x_{3}=0$ with increasing $x_{3}$ is large similarly to the earlier analyses ${ }^{[4,6,7]}$ and agrees with the experimental result ${ }^{[7]}$. In the case of Power law fluid for $R=50$, the stream lines starting at $x_{1}=0$ and $x_{3}=0$ cross at $x_{3}=$ $53.0^{\circ}, 46.2^{\circ}$ and $46.4^{\circ}$ for $x_{1}=0.5$ and $x_{2}=\pi / 2$ when 


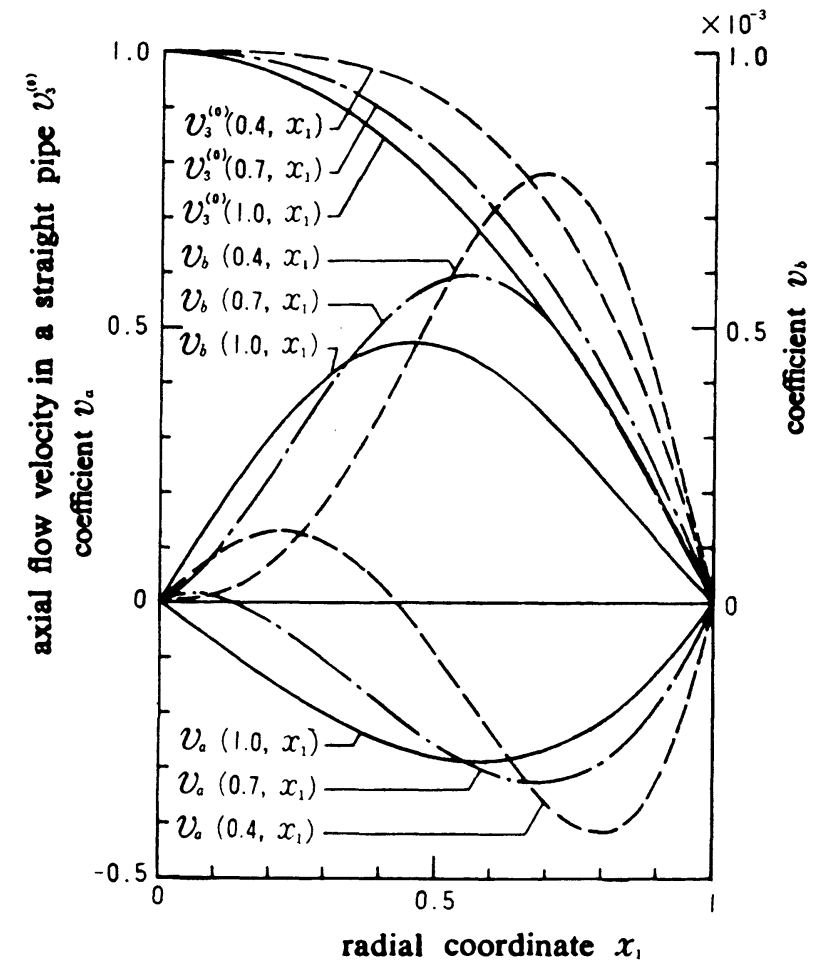

Fig. 4(a) Distributions of axial flow velocity $v_{3}{ }^{(0)}\left(n, x_{1}\right)$ in a straight pipe and coefficients $v_{a}\left(n, x_{1}\right)$ and $v_{b}\left(n, x_{1}\right)$

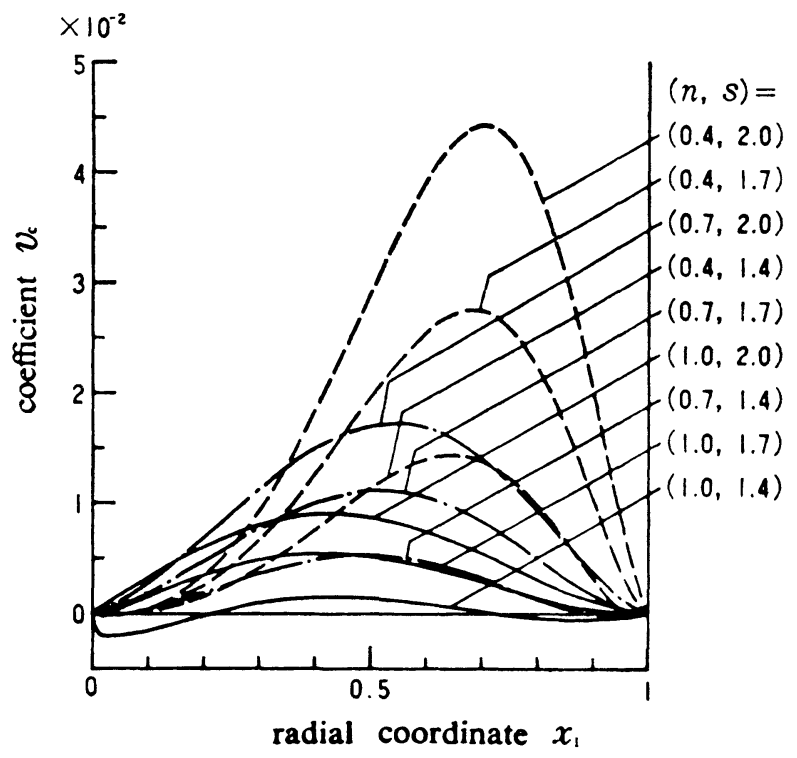

Fig. 4(b) Distributions of coefficient $v_{c}\left(n, s, x_{1}\right)$

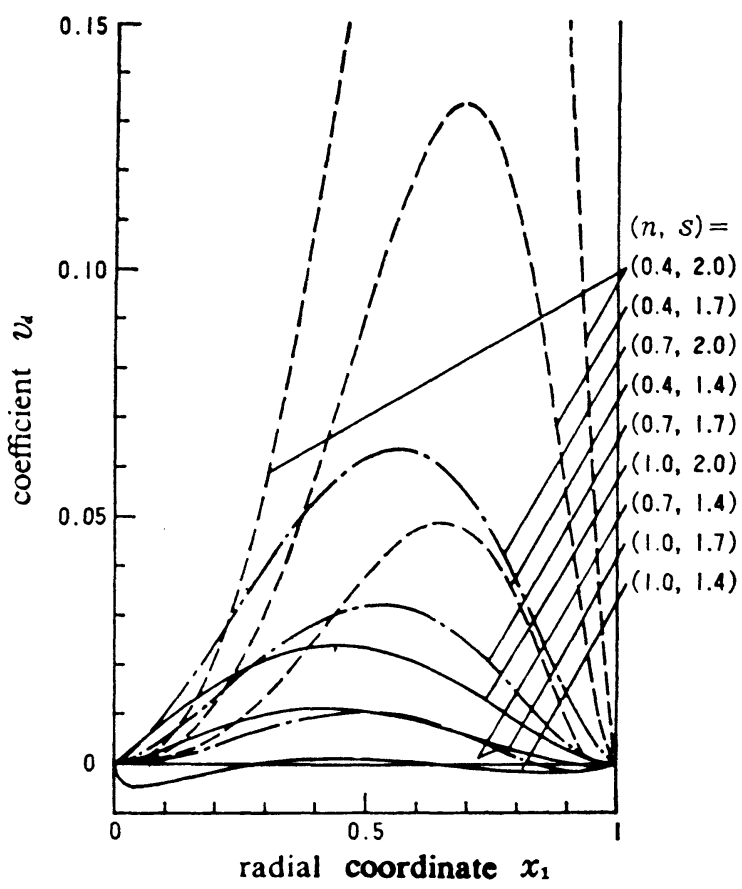

Fig. 4(c) Distributions of coefficient $v_{d}\left(n, s, x_{1}\right)$

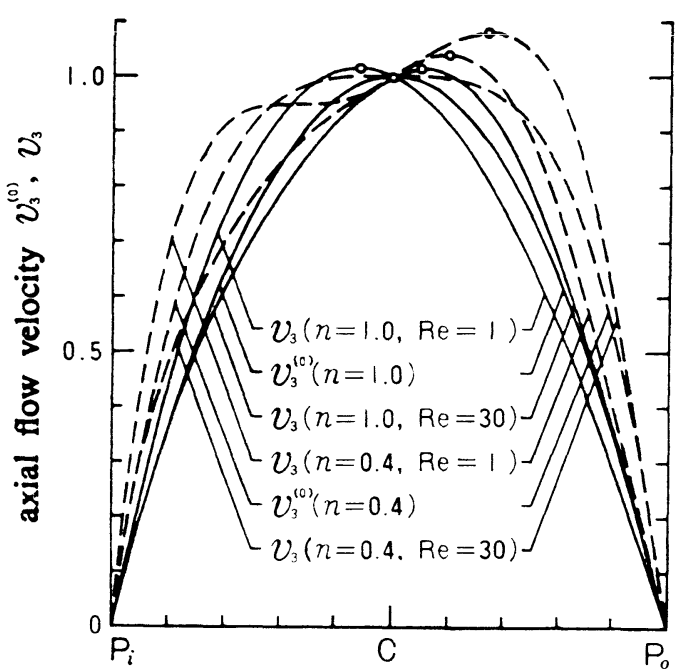

Fig. 5(a) Distributions of axial flow velocity $v_{3}{ }^{(0)}$ in a straight pipe, and axial flow velocity $v_{3}$ on the central plane for $R=3$ in the case of Power law fluid 


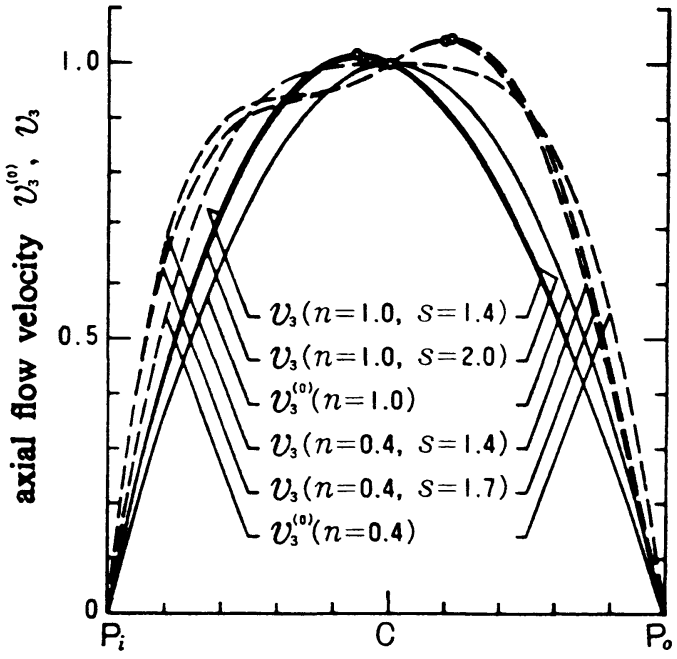

Fig. 5(b) Distributions of axial flow velocity $v_{3}(0)$ in a straight pipe, and axial flow velocity $v_{3}$ on the central plane for $R=3$ in case that $R e=1$ and $W e=1$

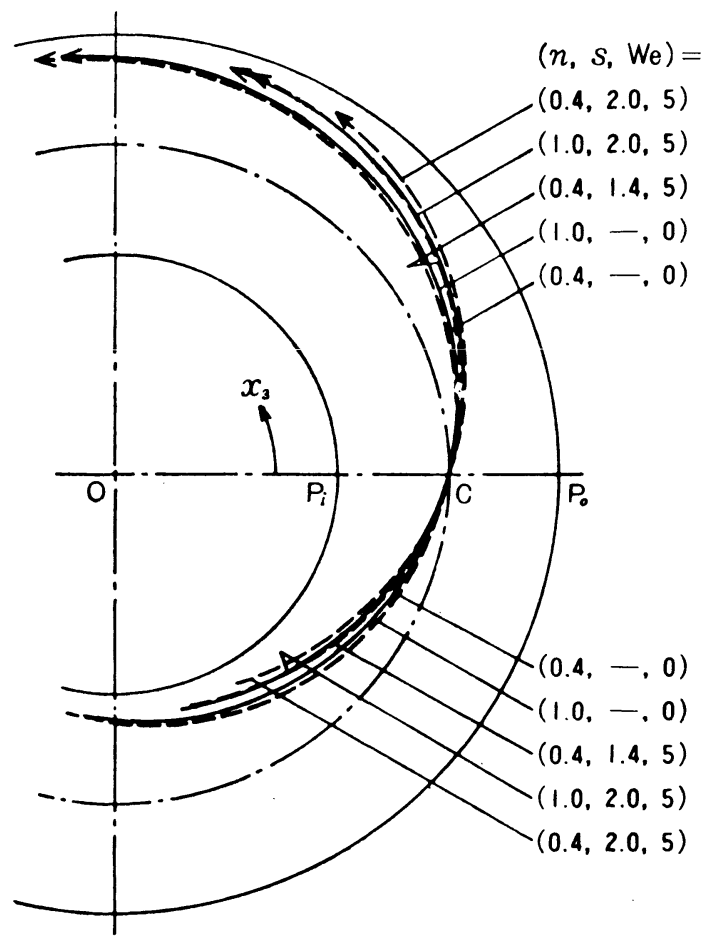

Fig. 6 Stream line on the central plane when $R=3$ and $R e=50$

$n=0.4,0.7$ and 1.0, respectively. For small $n$ the secondary flow is large, but the distribution of the axial flow velocity is gently-sloping in the vicinity of the pipe center. Therefore, the variation of the stream line on the central plane has not a simple tendency for the variation of $n$. On the other hand, the outward shift of the stream line on the central plane with increasing $x_{3}$ is large for large $s$ in the case of White-Metzner fluid, because the secondary flow increases with $s$.

The values of the radial flow velocity $v_{1}$ (eq. (31)) and the circumferential flow velocity $v_{2}$ (eq. (32)) are finite independent of the values of $s$ and $n$. However, the inexpedience exists that the axial flow velocity $v_{3}$ (eq. (33)) at the pipe center $x_{1}=0$ changes in relation to the circumferential angle $x_{2}$ when $s-n=0$. The inconsistency exists in the first term of $P\left(x_{1}\right)$ in eq. (34) for the isotropic pressure $p$ when $n \leqq 1 / 4$. Namely, $p$ is infinite at $x_{1}=0$ for $n<1 / 4$, and $p$ at $x_{1}=0$ is not isotropic for $n=1 / 4$ because it changes in relation to $x_{2}$. Moreover, the deviatric stress (eq. (36)) at $x_{1}=0$ is infinite for $n-1<0$ and/or $s-n-1<0$. The viscosity of real fluid is finite at zero shear-rate, but one of Power law fluid of $n<1$ is, infinite at zero shear-rate as stated in Chapter 1. The viscosity and/or the relaxation time of White-Metzner model are infinite at $x_{1}=0$ for $n-$ $1<0$ and/or $s-n-1<0$, respectively, because the second invariant of the rate-of-deformation tensor

$$
\begin{aligned}
M & \equiv 4\left|\Pi_{e}\right|=\left(\frac{n+1}{n}\right)^{2} x_{1}{ }^{\frac{2}{n}} \\
& -\frac{2}{R} \frac{n+1}{n} x_{1}^{\frac{1}{n}}\left(\frac{\partial v_{3}^{(1)}}{\partial x_{1}}-v_{3}^{(0)} \sin x_{2}\right)
\end{aligned}
$$

vanishes at $x_{1}=0$ in the present analysis. Hence, it is thought that the inconsistency appears in the values of the axial flow velocity, the isotropic pressure and the deviatric stress at the pipe center.

The viscosity and the normal stress coefficient of viscoelastic fluid like many polymer melts and solutions are constants independent of the shear-rate in the flow at low shearrate, but they decrease with the shear-rate in the flow at high shear-rate. Therefore, the White-Metzner model is applied to a flow at high shear-rate where the viscosity and the normal stress coefficient can be expressed by a power law for shear-rate. As the White-Metzner model can represent the Maxwell model, the viscosity and the normal stress coefficient of which are constants independent of shear-rate, the White-Metzner model can also indicate a flow at low shear-rate. However, because the White-Metzner model composed of four material constants alone can not represent decrements of the viscosity and the normal stress coefficient from constant values with increment of shear-rate, it can not be applied to a flow including from low to high shear-rate. Therefore, the White-Metzner model is meaningful to describe a flow at higher shear-rate than lower one as well as Power law fluid. In the present pipe flow there exists a region where the shear-rate (the second invariant of the rate-ofdeformation tensor) is very small, but the region is restricted to the vicinity of the pipe center for large characteristic shear-rate $W / a$. Hence, the present analysis can be applied 
to a flow of large characteristic shear-rate except the vicinity of the pipe center. Further, it can be applied in the entire region of the pipe when the characteristic shear-rate is small (the fluid is regarded as Maxwell fluid which has constant viscosity and constant relaxation time).

\section{Conclusion}

The steady flow of viscoelastic fluid in a toroidal pipe of circular cross-section is analytically solved with the WhiteMetzner constitutive equation by a perturbation method. The results are as follows:

(1) The flow rate of a secondary flow in the cross-sectional plane increases with decreasing the viscosity index $n$, and with increasing the elasticity index $s$, the Reynolds number $R e$ and the Weissenberg number $W e$.

(2) The position of the maximum axial flow velocity shifts on the central plane more outward from the pipe center for smaller $n$ and larger $R e$ in the case of Power law fluid, and for larger $s$ in the case of White-Metzner fluid.

(3) Owing to the centrifugal and elastic forces applied on the fluid, the stream line starting at a pipe center shifts outward on the central plane. The larger the values of $s, R e$ and $W e$ are, the larger the amount of the outward shift is.

Hence, the effects of $R e$ and $W e$ on the flow are shown to be similar to an available experimental result as well as earlier theoretical results. Also, it is found that the values $n$ and $s$, which represent the degrees of decrement of the fluid viscosity and the first normal stress coefficient with the shear-rate respectively, have significant effects on the flow at high shear-rate to which the earlier analyses could not be applied.
The White-Metzner model can be applied to a fluid, the viscous and elastic stresses of which are obedient to a power law for shear-rate, but not exactly to a flow including from low to high shear-rate. However, in case that the ratio of the flow velocity on the pipe center to the pipe radius is large, the velocity distribution of the flow can be obtained by the present analysis except in the very small region near the pipe center.

The authors would like to thank Mr. Yoshio Wada of Fukui University for assistance in preparing the manuscript.

\section{References}

[1] W. R. Dean; Phil. Mag., 4, No. 20, 208 (1927).

[2] W. R. Dean; Phil. Mag., 5, No. 30, 673 (1928).

[3] S. Murata, Y. Miyake, T. Inaba, H. Ogawa; Bull. J.S. M.E., 24, No. 188, 355 (1981).

[4] J. R. Jones; Quart. Journ. Mech. and Applied Math., 13, Pt. 4, 428 (1960).

[5] D. B. Clegg, G. Power; Appl. Sci. Res., Sect. A, 12, 199 (1963).

[6] R. H. Thomas, K. Walters; Journ. Fluid Mech., 16, 228 (1963).

[7] H. A. Barnes, K. Walters; Proc. Roy. Soc. Lond., Ser. A, 314, 85 (1969).

[8] J. L. White, A. B. Metzner; Journ. Appl. Polymer Sci., 7, 1867 (1963).

[9] M. M. Denn; Chem. Engg. Sci., 22, 395 (1967).

[10] Y. Tomita; Rheology (in Japanese), 398 (1975), Korona ed., Tokyo. 